\title{
Effect of heating and cooling combination therapy on patients with chronic low back pain: study protocol for a randomized controlled trial
}

\author{
Eun-Jung Kim', Young-Doo Choi ${ }^{1}$, Chi-Yeon Lim², Kyung-Ho Kim ${ }^{1}$ and Seung-Deok Lee 1,2,3*
}

\begin{abstract}
Background: Clinicians often apply heating or cooling stimulation for treatment of musculoskeletal pain. However, scalding, frostbite and skin ulcers may occur from the excessive use of either therapy alone. Heating and cooling combination therapy may be a suitable alternative for treatment of musculoskeletal diseases, although insufficient research has documented the safety and efficacy of such therapy. The purpose of this clinical trial is to determine the efficacy and safety of heating and cooling combination therapy for treatment of chronic low back pain.

Methods/Design: This is a multicenter, parallel-group, double-blinded, randomized controlled trial to evaluate the efficacy and safety of a heating and cooling combination therapeutic device (OCH-S100) in patients with chronic low back pain. Eighty participants with chronic low back pain will be recruited from two hospitals in South Korea (Dongguk University Ilsan Oriental Hospital and Dongguk University Bundang Oriental Hospital). Enrolled patients will be randomly divided into a treatment group and a sham group. Patients in both groups will be given 10 treatments (15 min per treatment) over 4 weeks. The protocol will consist of five cycles of heating/cooling therapy (maximum: $45^{\circ} \mathrm{C}$, minimum: $15^{\circ} \mathrm{C}$ ) in the treatment group, and five cycles of sham therapy (maximum: $1{ }^{\circ} \mathrm{C}$ above skin temperature, minimum: $1{ }^{\circ} \mathrm{C}$ below skin temperature) in the sham group. The primary outcome measure is change from baseline in the $100 \mathrm{~mm}$ Visual Analogue Scale (VAS) for pain after 4 weeks. There are six secondary outcome measures that consider disability or range of motion (ROM).
\end{abstract}

Discussion: This research will determine the efficacy and safety of heating and cooling combination therapy on chronic low back pain. The results of this trial may have important implications for the more widespread use of heating and cooling combination therapy for treatment of musculoskeletal pain.

Trial Registration: NCT02289170 (14 October 2014)

Keywords: Heating and cooling combination therapy, Low back pain, Randomized controlled trial (RCT)

\section{Background}

Heating and cooling therapies are widely used to treat musculoskeletal pain [1]. For example, in the United States, $75 \%$ of patients with low back pain are treated with heating therapy and $7 \%$ with cooling therapy [2].

Heating therapy increases tissue temperature, blood flow, metabolism and connective tissue extensibility [1]. There are also reports that heating therapy increases the activity in all of the Ia and many of the Ib afferents,

\footnotetext{
* Correspondence: chuckman100@gmail.com

${ }^{1}$ College of Korean Medicine, Dongguk University, Gyeongju, South Korea

${ }^{2}$ Department of Medicine, Dongguk University, Gyeongju, South Korea

Full list of author information is available at the end of the article
}

decreases the activity of most group II spindle afferents, decreases muscle spasms, and increases the speed of nerve conduction [3]. Other studies indicated that heating therapy is more effective than placebo in relieving pain, decreasing disability, decreasing muscle tension, and improving range of motion $[4,5]$.

There is evidence that cooling therapy decreases tissue blood flow due to vasoconstriction, and that it also reduces tissue metabolism, oxygen utilization, and inflammation $[1,6]$. Cooling therapy decreases the velocity of nerve conduction in superficial tissues by slowing the firing of muscle spindle afferents and reflex responses, thus decreasing muscle spasms and pain [7]. Use of 
cooling therapy for musculoskeletal problems can also reduce intake of painkillers because it reduces pain and body fluid penetration [8].

However, the overuse of cooling therapy or heating therapy due to physiological adaptation can lead to skin damage such as scalding, frostbite, and ulcers $[1,6]$. We developed a heating and cooling combination therapy device $(\mathrm{OCH}-\mathrm{S} 00)$ that reduces the probability of skin damage. This device maximizes the stimulation and reduces the potential harm from heating and cooling by use of a probe to reduce sensory adaptation.

Thermo-receptors are sensitive to different temperatures. Thus, TRPA1 is mainly responsible for painful cold sensation below $18{ }^{\circ} \mathrm{C}$, TRPM8 for cool sensation at 23 to $28{ }^{\circ} \mathrm{C}$, TRPV2 for warm sensation at 30 to $39^{\circ} \mathrm{C}$, and TRPV1 for painful hot sensation above $43{ }^{\circ} \mathrm{C}$ [9]. The heating and cooling device described here stimulates the cold, cool, warm and hot receptors by applying temperatures in the range of 15 to $45^{\circ} \mathrm{C}$, well below the burn point. Thus, this device is expected to have antiinflammatory effects, increase muscle relaxation, decrease edema and provide pain relief, without damaging the skin.

The purpose of this research is to evaluate the safety and efficacy of the OCH-S100 heating and cooling combination therapy device on patients with chronic low back pain.

\section{Methods/Design Study design}

This research of the clinical efficacy and safety of heating and cooling combination therapy is a multicenter, randomized, double-blinded, parallel-group study. All enrolled subjects are patients with chronic low back pain. This research protocol was externally peer reviewed by Korean Ministry of Food and Drug Safety (KMFDS) and Korean Health Industry Development Institute (KHIDI). Two clinical research centers in Korea will participate in this trial: Dongguk University Ilsan Oriental Hospital and Dongguk University Bundang Oriental Hospital. This research protocol was approved by Dongguk University Ilsan Oriental Hospital's Institutional Review board (IRB) and Dongguk University Bundang Oriental Hospital's IRB. Each subject will provide informed consent before enrollment.

\section{Recruitment}

Participants will be recruited through advertisements in local newspapers and on bulletin boards at each trial center. The target sample size is 80 subjects. Telephone screening will be performed before the trial to determine if subjects fulfill the selection criteria. Final enrollment will be determined by an orthopedist and a radiologist through physical exams, radiological tests, blood and urine tests, and relevant questionnaires.

\section{Participants}

All outpatients who are 20 to 75 years of age and have suffered from low back pain in the previous 3 months or more and had ratings over $40 \mathrm{~mm}$ on a $100 \mathrm{~mm}$ Visual Analog Scale (VAS) for pain will be screened. Subjects will be excluded if any of the following are present: (i) trauma or surgery in the lumbar region in the 6 months prior to enrollment; (ii) low back pain accompanied by sciatica; (iii) systemic disease or severe dysfunction due to another medical problem (cancer, infection, etcetera); (iv) history of using medication(s), traditional oriental medical care, or physiotherapy for treatment of low back pain in the 4 weeks prior to enrollment; (v) neurological disease, such as topical or whole body hypoesthesia, that may affect temperature sensation (stroke, peripheral neuropathy, etcetera); (vi) hypersensitivity to heating and cooling stimulation; (vii) pregnancy or lactating; or (viii) participation in other clinical trials in the 3 months prior to or after enrolling in this trial.

\section{Randomization and blinding}

Randomization will be performed by an independent statistician using a computerized random-number generator through the block-randomization method of SAS version 9.1 (SAS Institute Inc. Cary, NC, USA). Separate randomization files will be created for each study site. Allocation concealment will be maintained by use of opaque sealed envelopes. After the researcher screens a patient for inclusion and receives signed informed consent, the next envelope in the sequence will be accessed and opened by the certified Korean Medicine Doctor who supervises the medical device, immediately prior to treatment. A total of 80 participants will be randomly and blindly assigned to a treatment group or a sham group. All subjects, practitioners, and the outcome assessor will be blinded to group assignment. Blinding of the certified Korean Medicine Doctor who supervises the medical device and sets up the treatment (medical equipment manager) is not possible. The patients will be told: "You will be treated with one of two heating and cooling combination treatments. One has a large range of temperature change and the other has a small range of temperature change."

\section{Treatment and assessment}

Subjects in both groups will be treated 10 times over 4 weeks. The treatment term of 4 weeks was based on previous studies of the treatment of general chronic low back pain [10]. Each treatment will be $15 \mathrm{~min}$ and there will be two to three treatments per week, based on the 
protocol of previous research on heating and cooling combination treatment [11]. The outcome assessor (who does not participate in the treatment) will measure the degree of pain sensitivity by pressing eight acupoints (four on each side of the waist; BL23, BL24, BL25, and BL26) that are frequently used to assess low back pain [12-16]. This assessment will employ a pressure algometer, with a circular and flat probe of $1 \mathrm{~cm}$ diameter (Commander Algometer, JTECH Medical, Midvale, Utah, USA), to identify the two most painful acupoints. Before treatment, the medical equipment manager will measure the skin temperature of each patient in the treatment and sham groups using thermometer (Superfast Thermapen Pro-surface, ETI, England) (Table 1).

\section{Heating and cooling combination therapy}

The patients in this group will receive heating and cooling combination therapy. The probe is attached to the acupuncture points for $15 \mathrm{~min}$, and its temperature will be cycled five times, from the maximum of $45^{\circ} \mathrm{C}$ to the minimum of $15{ }^{\circ} \mathrm{C}$. This temperature range was chosen to provide stimulation of cold, cool, warm and hot receptors.

\section{Sham therapy}

The patients in this group will receive sham heating and cooling combination therapy under identical conditions, except for probe temperature. The probe's temperature will be cycled five times, from $1{ }^{\circ} \mathrm{C}$ above skin temperature to $1{ }^{\circ} \mathrm{C}$ below skin temperature.

During the 4-week study, patients will be prohibited from using any treatment that could influence the results, such as analgesics, nonsteroidal anti-inflammatory drugs, muscle relaxants, oral corticosteroids, antidepressants, steroid injections, high frequency heat coagulation, physiotherapy, acupuncture, moxibustion and surgery.

Table 1 Content of outcome measurement

\begin{tabular}{|c|c|c|c|c|c|c|c|c|c|c|c|c|}
\hline \multirow[b]{2}{*}{ Outcome measure } & \multicolumn{12}{|c|}{ Treatment Number } \\
\hline & $\mathrm{B}$ & 1 & 2 & 3 & 4 & 5 & 6 & 7 & 8 & 9 & 10 & $\mathrm{~F}$ \\
\hline \multicolumn{13}{|l|}{ Primary outcome } \\
\hline VAS & $x$ & $x$ & $x$ & $x$ & $x$ & $x$ & $x$ & $x$ & $x$ & $x$ & $x$ & $x$ \\
\hline \multicolumn{13}{|l|}{ Secondary outcomes } \\
\hline KODI & & $x$ & & & & & $x$ & & & & $x$ & $x$ \\
\hline KRMDQ & & $x$ & & & & & $x$ & & & & $x$ & $x$ \\
\hline TMBQ & & $x$ & & & & & $x$ & & & & $x$ & $x$ \\
\hline Modified Schöber test & & $x$ & & & & & $x$ & & & & $x$ & $x$ \\
\hline Finger-to-floor distance & & $x$ & & & & & $x$ & & & & $x$ & $x$ \\
\hline Finger-to thigh distance & & $x$ & & & & & $x$ & & & & $x$ & $x$ \\
\hline
\end{tabular}

$B$ baseline, $F$ follow-up, VAS Visual Analog Scale, KODI Korean Oswestry disability index, KRMDQ Korean Roland Morris Disability Questionnaire, $T M B Q$ Traditional Medicine Back pain Questionnaire
Patients who use any of these treatments will be excluded. All patients will be questioned about medication usage during each treatment session.

\section{Quality assurance}

We will educate all practitioners and set qualification standards to make sure the patients are treated with high standards and in accordance with the trial protocol.

Treatment will be administered by doctors of Korean Medicine who have extensive clinical experience with heating and cooling stimulation therapy and moxibustion and the following qualifications: (i) graduate of a 6-year full-time course in Korean Medicine, taught as a college program; (ii) certified by the Korean Ministry of Health and Welfare as a Korean Medicine Doctor; (iii) more than 1 year of postgraduate clinical training in an Oriental medicine hospital; and (iv) completion of the first-year residency program in our Department of Acupuncture and Moxibustion.

Practitioners and medical equipment managers will attend a 2-day training workshop about the treatment programs and will be educated on selection of acupoints by using a pressure algometer. Medical equipment managers will also be educated on measuring skin temperature and setting the treatment and sham devices. A written protocol and standardized recording documents will be provided. Because the relationship between a practitioner and patient could influence the results, all such interactions will be strictly limited, except when reporting side effects. All practitioners and outcome assessors will be blinded to group allocations, and medical equipment managers will not participate in the interventions. To assure blinding of patients, we will put a small fabric curtain next to the device to block the patient's view.

\section{Analysis of data}

A VAS will be used to assess the effect of treatment. The primary endpoint is the change in the $100 \mathrm{~mm}$ Pain VAS from baseline (before treatment) to 4 weeks $[10,12,17]$. The $100 \mathrm{~mm}$ VAS is widely used because it provides reliable and valid measurements of pain intensity [18]. Each patient will be asked to check their pain intensity along an ungraded $100 \mathrm{~mm}$ horizontal line, where ' 0 ' represents 'no pain' and ' 100 ' represents 'unbearable pain'.

The secondary endpoints are change from baseline in three evaluations of functional disability (Korean Oswestry disability index [KODI], Korean Roland Morris Disability Questionnaire [KRMDQ], and Traditional Medicine Back pain Questionnaire [TMBQ]) and three evaluations of range of motion (ROM) (modified Schöber test, finger-to-floor distance, and finger-to-thigh distance). These parameters will be measured at baseline, 2 weeks, 4 weeks, and at 4 weeks after the last treatment. 
The KODI has 10 criteria related to daily living activities and each item has six possible responses, ranging from 'no problem' to 'not possible'. The KODI focuses on a limitation in the range of physical functions, including standing, walking, lifting, sitting, lying down, dressing, and personal care [18]. We excluded questions about sexual activities because many subjects do not provide honest answers about their sex lives, and it is hard to account for subjects who are single. The KRMDQ contains 24 yes/no queries and also focuses on limitations in physical functions, including walking, bending over, sitting, lying down, dressing, sleeping, selfcare, and daily activities. The KRMDQ is easy to understand, interpret and score. Higher scores in the KRMDQ and KODI indicate greater disability [18]. We will use Korean versions of these surveys, which have documented reliability and validity $[19,20]$. The TMBQ evaluates low back pain and is based on traditional Korean medicine [21].

The modified Schöber test measures the curvature of the spine. In this test, the lumbosacral junction is marked on the back; a second mark is placed $5 \mathrm{~cm}$ below the junction, and a third mark is placed $10 \mathrm{~cm}$ above the junction. Then the subject is asked to bend forward as far as possible, and the new distance between the second and third marks is measured [22]. We will also use a digital flexibility testing device (Standing Trunk Flexion meter TKK5403, Takei Co., Japan) to assess the trunk flexibility, finger-to-floor distance (distance between the fingertips and the floor after forward flexion with knees straight, while on a $20-\mathrm{cm}$ high box) [23], and finger-to-thigh distance (distance between the fingertips and the floor after lateral bending with knees straight, while on a 20-cm high box) [24].

\section{Safety assessments}

Any physical or clinical changes will be reported by the assessor, practitioner, and patient at each visit. All symptoms, date of onset, and duration will be recorded.

\section{Sample size}

The primary endpoint will be change in $100 \mathrm{~mm}$ VAS score after 4 weeks of treatment. Liu et al. [25] previously showed that the 4-week change was 7.6 for the test device and 3.9 for the control device. Patients will be randomized to the test and sham groups in a 1:1 ratio. Assuming a reference standard deviation (SD) of 5.37, power analysis indicates that a sample size of 34 has an $80 \%$ probability of yielding a significant difference at the level of 0.05 . In order to achieve stronger results, a onesided $97.5 \%$ lower confidence limit for the treatment difference (VAS on test device - VAS on sham device) will be greater than 0 . We will allow for a $15 \%$ dropout rate by randomization of 40 subjects to each group.

\section{Statistical analysis}

All data will be analyzed using SAS version 9.1 (SAS institute Inc. Cary, NC, USA). All statistical tests will be two-sided, and the level of significance will be set at 0.05 . Continuous variables will be expressed as means \pm SDs and categorical variables as numbers and percentages. For analysis of the primary endpoint, the two-sided $95 \%$ confidence interval will calculated by analysis of covariance (ANCOVA) and the model will include fixed-effect for study site. Student's $t$-test will be used for evaluation of primary and secondary end-points. Categorical data will be analyzed using Pearson's chisquare test or Fisher's exact test, as appropriate.

For safety analysis, the numbers and percentages of patients will be presented in a frequency table. Patients with adverse events will be listed along with their medical histories and concomitant medications, with start and stop dates. Categorical variables will be summarized by frequency tables, and continuous laboratory variables, and changes from baseline will be expressed by summary statistics. In addition, the number and percentage of patients with each value below, within and above the normal range will be tabulated, and "shift tables" (summaries of individual changes in laboratory parameters) will be generated.

\section{Discussion}

This clinical research aims to evaluate the safety and efficacy of OCH-S100, a device designed to administer heating and cooling combination therapy and provide relief for patients with chronic low back pain.

Heating and cooling therapy is widely used for treatment of musculoskeletal pain in clinical practice $[7,11]$. The beneficial effects of this therapy may be mediated by changing blood flow, decreasing inflammation, induction of contraction and relaxation of blood vessels, decreasing edema, or decreasing stiffness of the muscle [11], but the underlying mechanism has not yet been proven. Moreover, there is only limited research on the efficacy of heating and cooling combination therapy. Thus, the clinical research described here is one of the few studies to test rigorously the efficacy and safety of heating and cooling combination therapy. Our research was designed to be rigorous, in that it will employ two study centers, double blinding of all assessors and practitioners, prior education of all practitioners, and fabric curtains to block patients' view of the device. Moreover, a certified Korean Medicine Doctor with extensive experience will be treating all patients.

The device used in this research can precisely deliver temperatures from $15{ }^{\circ} \mathrm{C}$ to $45{ }^{\circ} \mathrm{C}$, temperatures that do not burn or damage the skin. Therefore, this research will be able to document the effect of stimulation of the 
cold, cool, warm, and hot receptors on patients with chronic low back pain.

\section{Trial status}

Patient recruitment is ongoing. Enrollment and trial completion is expected by the end of 2015 .

\section{Abbreviations}

ANCOVA: analysis of covariance; IRB: Institutional Review Board; KODI: Korean Oswestry Disability Index; KRMDQ: Korean Roland Morris Disability Questionnaire; ROM: range of motion; SD: standard deviation; TMBQ: Traditional Medicine Back pain Questionnaire; VAS: visual analog scale.

\section{Competing interests}

The authors declare that they have no competing interests.

\section{Authors' contributions}

KHK, SDL and EJK conceived the project and formulated and coordinated its execution. YDC substantially contributed to conducting the clinical trial, participated in its design, and helped draft the manuscript. EJK wrote the first draft of the manuscript. CYL provided statistical advice, participated in its design and wrote the relevant sections of the manuscript. KHK and SDL revised the manuscript. All authors read and approved the submitted version of the manuscript.

\section{Acknowledgments}

This study was supported by a grant from the Traditional Korean Medicine R\&D Project, Ministry of Health \&Welfare, Republic of Korea (HI11C2135)

\section{Author details}

${ }^{1}$ College of Korean Medicine, Dongguk University, Gyeongju, South Korea ${ }^{2}$ Department of Medicine, Dongguk University, Gyeongju, South Korea. ${ }^{3}$ Department of Acupuncture and Moxibustion, Dongguk University International Hospital, Siksa-dong, Ilsandong-gu, Goyang, Gyeonggi-do, Korea

Received: 6 April 2015 Accepted: 8 June 2015

Published online: 26 June 2015

\section{References}

1. Nadler SF, Weingand K, Kruse RJ. The physiologic basis and clinica applications of cryotherapy and thermotherapy for the pain practitioner. Pain Physician. 2004;7:395-9.

2. Deyo RA, Tsui-Wu YJ. Descriptive epidemiology of low-back pain and its related medical care in the United States. Spine (Phila Pa 1976). 1987;12:264-8.

3. Mense S. Effects of temperature on the discharges of muscle spindles and tendon organs. Pflugers Arch. 1978;374:159-66.

4. Nadler SF, Steiner DJ, Erasala GN, Hengehold DA, Abeln SB, Weingand KW Continuous low-level heatwrap therapy for treating acute nonspecific low back pain. Arch Phys Med Rehabil. 2003;84:329-34.

5. Nadler SF, Steiner DJ, Petty SR, Erasala GN, Hengehold DA, Weingand KW. Overnight use of continuous low-level heatwrap therapy for relief of low back pain. Arch Phys Med Rehabil. 2003:84:335-42.

6. Ciolek JJ. Cryotherapy. Review of physiological effects and clinical application. Cleve Clin Q. 1985:52:193-201.

7. Cochrane DJ. Alternating hot and cold water immersion for athlete recovery: a review. Phys Ther Sport. 2004:5:26-32.

8. Meeusen R, Handelberg F, Framhout L, Daems S. The influence of cryotherapy (Cryotron ${ }^{\oplus}$ ) on pain and inflammation following arthroscopy of the shoulder. Kinesitherapie scientifique. 2004;450:11-7.

9. Wang H, Woolf CJ. Pain TRPs. Neuron. 2005;46:9-12.

10. Molsberger AF, Mau J, Pawelec DB, Winkler J. Does acupuncture improve the orthopedic management of chronic low back pain-a randomized, blinded, controlled trial with 3 months follow up. Pain. 2002;99:579-87.

11. Hing WA, White SG, Bouaaphone A, Lee P. Contrast therapy-a systematic review. Phys Ther Sport. 2008;9:148-61.

12. Zaringhalam J, Manaheji H, Rastqar A, Zaringhalam M. Reduction of chronic non-specific low back pain: a randomised controlled clinical trial on acupuncture and baclofen. Chin Med. 2010;5:15.
13. Cherkin DC, Sherman KJ, Avins AL, Erro JH, Ichikawa L, Barlow WE, et al. A randomized trial comparing acupuncture, simulated acupuncture, and usual care for chronic low back pain. Arch Intern Med. 2009;169:858-66.

14. Brinkhaus B, Witt CM, Jena S, Linde K, Streng A, Wagenpfeil S, et al. Acupuncture in patients with chronic low back pain: a randomized controlled trial. Arch Intern Med. 2006:166:450-7.

15. Tsui ML, Cheing GL. The effectiveness of electroacupuncture versus electrical heat acupuncture in the management of chronic low-back pain. J Altern Complement Med. 2004;10:803-9.

16. Meng CF, Wang D, Ngeow J, Lao L, Peterson M, Paget S. Acupuncture for chronic low back pain in older patients: a randomized, controlled trial. Rheumatology (Oxford). 2003:42:1508-17.

17. Kerr DP, Walsh DM, Baxter D. Acupuncture in the management of chronic low back pain: a blinded randomized controlled trial. Clin J Pain. 2003;19:364-70.

18. Ostelo RW, de Vet HC. Clinically important outcomes in low back pain. Best Pract Res Clin Rheumatol. 2005:19:593-607.

19. Kim DY, Lee SH, Lee HY, Lee HJ, Chang SB, Chung SK, et al. Validation of the Korean version of the oswestry disability index. Spine (Phila Pa 1976). 2005:30:E123-7.

20. Lee JS, Lee DH, Suh KT, Kim JI, Lim JM, Goh TS. Validation of the Korean version of the Roland-Morris Disability Questionnaire. Eur Spine J. 2011;20:2115-9.

21. Biopharmaceuticals and Herbal Medicine Evaluation Department KFDA. Clinical trial guidelines of herbal medicine drug - Chronic low back pain. In: Herbal Medicinal Products Division, Biopharmaceuticals and Herbal Medicine Evaluation Department:Korea Food \& Drug Administration. 1st ed. 2010.

22. Moll JM, Wright V. Normal range of spinal mobility. An objective clinical study. Ann Rheum Dis. 1971;30:381-6.

23. Perret C, Poiraudeau S, Fermanian J, Colau MM, Benhamou MA, Revel M. Validity, reliability, and responsiveness of the fingertip-to-floor test. Arch Phys Med Rehabil. 2001:82:1566-70.

24. Schiltenwolf M, Buchner M, Heindl B, von Reumont J, Muller A, Eich W. Comparison of a biopsychosocial therapy (BT) with a conventional biomedical therapy (MT) of subacute low back pain in the first episode of sick leave: a randomized controlled trial. Eur Spine J. 2006:15:1083-92.

25. Xiaoguang Liu JL, Timon Chengyi L, Jianqin Y, Qingming L. Randomized trial comparing exercise therapy, alternating cold and hot therapy, and low intensity laser therapy for chronic lumbar muscle strain. In: International Society for Optics and Photonics (In Photonics and Optoelectronics Meetings). 2008. p. $72801 B$.

\section{Submit your next manuscript to BioMed Central and take full advantage of:}

- Convenient online submission

- Thorough peer review

- No space constraints or color figure charges

- Immediate publication on acceptance

- Inclusion in PubMed, CAS, Scopus and Google Scholar

- Research which is freely available for redistribution 